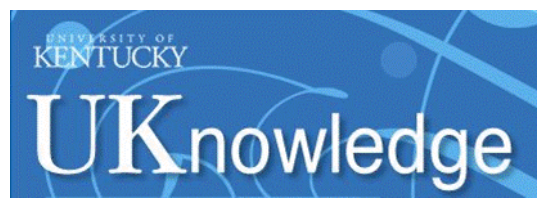

University of Kentucky

UKnowledge

10-27-2016

\title{
The Intermediate-Line Region in Active Galactic Nuclei
}

T. P. Adhikari

Polish Academy of Sciences, Poland

A. Różańska

Polish Academy of Sciences, Poland

B. Czerny

Polish Academy of Sciences, Poland

K. Hryniewicz

Polish Academy of Sciences, Poland

Gary J. Ferland

University of Kentucky, gary@uky.edu

Follow this and additional works at: https://uknowledge.uky.edu/physastron_facpub

Part of the Astrophysics and Astronomy Commons, and the Physics Commons

Right click to open a feedback form in a new tab to let us know how this document benefits you.

\section{Repository Citation}

Adhikari, T. P.; Różańska, A.; Czerny, B.; Hryniewicz, K.; and Ferland, Gary J., "The Intermediate-Line Region in Active Galactic Nuclei" (2016). Physics and Astronomy Faculty Publications. 407.

https://uknowledge.uky.edu/physastron_facpub/407

This Article is brought to you for free and open access by the Physics and Astronomy at UKnowledge. It has been accepted for inclusion in Physics and Astronomy Faculty Publications by an authorized administrator of UKnowledge. For more information, please contact UKnowledge@lsv.uky.edu. 


\section{The Intermediate-Line Region in Active Galactic Nuclei}

Digital Object Identifier (DOI)

https://doi.org/10.3847/0004-637X/831/1/68

\section{Notes/Citation Information}

Published in The Astrophysical Journal, v. 831, no. 1, 68, p. 1-9.

(c) 2016. The American Astronomical Society. All rights reserved.

The copyright holder has granted the permission for posting the article here. 


\title{
THE INTERMEDIATE-LINE REGION IN ACTIVE GALACTIC NUCLEI
}

\author{
T. P. AdHIKAri ${ }^{1}$, A. Różańska ${ }^{1}$, B. Czerny ${ }^{2}$, K. Hryniewicz ${ }^{1}$, And G. J. Ferland ${ }^{1,3}$ \\ ${ }^{1}$ Nicolaus Copernicus Astronomical Center, Polish Academy of Sciences, Bartycka 18, 00-716, Warsaw, Poland; tek@camk.edu.pl \\ ${ }^{2}$ Center for Theoretical Physics, Polish Academy of Sciences, Aleja Lotnikow 32/46, Warsaw, Poland \\ ${ }^{3}$ Department of Physics and Astronomy, The University of Kentucky, Lexington, KY 40506, USA \\ Received 2016 May 29; revised 2016 August 10; accepted 2016 August 12; published 2016 October 27
}

\begin{abstract}
We show that the recently observed suppression of the gap between the broad-line region (BLR) and the narrowline region (NLR) in some active galactic nuclei (AGNs) can be fully explained by an increase of the gas density in the emitting region. Our model predicts the formation of the intermediate-line region (ILR) that is observed in some Seyfert galaxies by the detection of emission lines with intermediate-velocity FWHM $\sim 700-1200 \mathrm{~km} \mathrm{~s}^{-1}$. These lines are believed to be originating from an ILR located somewhere between the BLR and NLR. As was previously proved, the apparent gap is assumed to be caused by the presence of dust beyond the sublimation radius. Our computations with the use of the CLOUDY photoionization code show that the differences in the shape of the spectral energy distribution from the central region of AGNs do not diminish the apparent gap in the line emission in those objects. A strong discontinuity in the line emission versus radius exists for all lines at the dust sublimation radius. However, increasing the gas density to $\sim 10^{11.5} \mathrm{~cm}^{-3}$ at the sublimation radius provides the continuous line emission versus radius and fully explains the recently observed lack of apparent gap in some AGNs. We show that such a high density is consistent with the density of upper layers of an accretion disk atmosphere. Therefore, the upper layers of the disk atmosphere can give rise to the formation of observed emission-line clouds.
\end{abstract}

Key words: galaxies: active - galaxies: individual (NLR-BLR) - methods: numerical - quasars: emission lines radiative transfer

\section{INTRODUCTION}

Observations of active galactic nuclei (AGNs) clearly show different types of emission lines in their spectra. Depending on the width of the emitted lines, they are classified as narrow lines, with FWHM $\sim 500 \mathrm{~km} \mathrm{~s}^{-1}$, or broad lines, with FWHM $\gtrsim 2000 \mathrm{~km} \mathrm{~s}^{-1}$. This implies two different regions of line formation, i.e., the narrow-line region (NLR) and the broad-line region (BLR), respectively. Although the physical conditions of these regions are quite well understood (Davidson 1972; Krolik et al. 1981; Blandford et al. 1990; Pier \& Voit 1995; Dopita et al. 2002; Groves et al. 2004a, 2004b; Czerny \& Hryniewicz 2011; Baskin et al. 2014; Stern et al. 2014; Czerny et al. 2015; Stern et al. 2016), there are still several issues to be explored. One of those problems is the significant suppression of the line emission in a region between the BLR and NLR in most AGNs (Boroson \& Green 1992; Osterbrock \& Ferland 2006). A leading explanation for this feature is the dust suppression introduced by Netzer \& Laor (1993, hereafter NL93). On the other hand, recent observations of several objects indicate the existence of an intermediate-line emission region (ILR), between the BLR and NLR, which produces emission lines with velocity FWHM $700-1200 \mathrm{~km} \mathrm{~s}^{-1}$. Using the statistical investigations of broad UV lines in QSOs, Brotherton et al. (1994) discussed the ILR as an inner extension of the NLR. Mason et al. (1996) found evidence for an ILR with velocity FWHM $\sim 1000 \mathrm{~km} \mathrm{~s}^{-1}$ that produces a significant amount of both permitted and forbidden line fluxes in NLSy1 RE J1034+396. For the Sy1 NGC 4151, Crenshaw \& Kraemer (2007) identified a line emission component with width FWHM $=1170 \mathrm{~km} \mathrm{~s}^{-1}$, most probably originating between the BLR and NLR. Detailed spectral analysis of a large number of Sloan Digital Sky Survey (SDSS) sources has revealed the presence of an intermediate component of line emission with velocity width in between that of broad and narrow components (Hu et al. 2008a, 2008b; Zhu et al. 2009). Additionally, Crenshaw et al. (2009) detected ILR emission with a width of $680 \mathrm{~km} \mathrm{~s}^{-1}$ in the historically low state spectrum of NGC 5548 obtained with the Space Telescope Imaging Spectrograph on the Hubble Space Telescope (HST). Using the HST/FOS spectra of quasar O I $287, \mathrm{Li}$ et al. (2015) reported the detection of intermediatewidth emission lines originating at a distance of $\sim 2.9 \mathrm{pc}$ from the central black hole. The following questions remain unanswered: Is there any separation between gas responsible for broad- and narrow-line emission in different types of AGNs? What is the physical mechanism leading to the existence of this gap in some sources, and why is the gap not observed in all of them?

Photoionization calculations provide a powerful tool to model the line emission from gas clouds powered by the continuum radiation from the nucleus. Detailed analysis has shown that BLR clouds are denser and located closer to the AGN center, while the NLR clouds have lower density and are more distant (see for details Blandford et al. 1990; Osterbrock $\&$ Ferland 2006). Nevertheless, the lack of significant line emission from the intermediate zone between the BLR and NLR is not naturally explained by photoionization models.

The presence of dust complicates the physics by introducing additional processes (Ferland \& Netzer 1979; Baldwin et al. 1991; van Hoof et al. 2004, and references therein) in the radiation-matter interactions, which have to be treated properly in simulations of the gas emission. Observations have shown the existence of dust in the NLR for almost all types of AGNs (De Robertis \& Osterbrock 1984; Ferland \& Osterbrock 1986; Wills et al. 1993). However, it has been argued that the BLR clouds are devoid of dust since there is a lack of depletion of refractory elements in the gaseous phase (Gaskell et al. 1981). It is commonly believed that the radiation 
energy is so high in the BLR that the dust, if present, sublimes and no longer survives there (Czerny \& Hryniewicz 2011). Reverberation mapping studies show that the BLR clouds are located at a distance smaller by a factor of 4 to 5 than the hot dust emission (Suganuma et al. 2006; Koshida et al. 2014). On the other hand, Nenkova et al. (2008) have shown that the closest region where dust can survive in the full radiation field is the face of the dusty torus, located at approximately $0.4 \mathrm{pc}$ for AGNs of typical luminosity (see their Equation (1)). While Equation (1) of Nenkova et al. (2008) gives the smallest radius at which the dust absorption coefficient reflects the full grain mixture, the largest grains survive to closer radii, where they are presumably detected by the reverberation measurements.

The observed apparent gap in the line emission region between the BLR and NLR was explained by the dust content in NLR clouds by NL93. The authors calculated emission from a continuous radial distribution of clouds extending from the BLR to the NLR, using the numerical photoionization code ION (Rees et al. 1989). Their assumption was that the dust is present in NLR but sublimes in a higher ionization region, around the BLR. The authors have shown that the reduced line emission versus radius is a result of the dust extinction of ionizing radiation, as well as the dust destruction of the line photons. The dust absorption becomes more efficient with decreasing radial distance from the nucleus and gives rise to an empty intermediate region, where gas is present but the line emission is heavily suppressed. The dust fully sublimates at smaller radii, and line emission increases dramatically, by about an order of magnitude, giving rise to the BLR. NL93 successfully demonstrated the apparent gap in the line emission versus radius, although their result was obtained for a particular spectral energy distribution (SED) typical for Sy1 AGNs (Mathews \& Ferland 1987), and for a specific value of the density $n_{\mathrm{H}}=10^{9.4} \mathrm{~cm}^{-3}$ at the sublimation radius at $\sim 0.1 \mathrm{pc}$.

The aim of this paper is to explain the disappearance of an apparent gap in line emission seen for some sources within the framework of the NL93 model. We use the photoionization code CLOUDY, version 13.03 (Ferland et al. 2013), to determine line luminosities for different shapes of the incident radiation representative for various subclasses of AGNs, as measured from recent observations: Sy1.5 galaxy Mrk 509 (Kaastra et al. 2011), Sy1 galaxy NGC 5548 (Mehdipour et al. 2015), and NLSy1 galaxy PMN J0948+0022 (D'Ammando et al. 2015). In addition, we also use a flexible parametric shape of the SED as a Band function (Band et al. 1993). All our results presented below focus on the five major emission lines usually detected in those objects: $\mathrm{H} \beta \lambda 4861.36$, He II $\lambda 1640.00, \quad M g$ II $\quad \lambda 2798.0, \quad$ C III] $\lambda 1909.00, \quad$ and [O III] $\lambda 5006.84$.

We show that the various shapes of the SED used in our computations with the model parameters taken from NL93 do not remove the apparent gap in the line emission versus radius. For lines considered by us, we always obtain an apparent gap similar to the result of NL93. Therefore, the observed properties of the ILR cannot be explained only by considering the different shapes of radiation illuminating the gas and dust in our simulations. However, increasing the gas density at the sublimation radius yields to the continuous line emission versus radius and fully explains the observed emission from the ILR.

The structure of this paper is as follows: Section 2 describes the parameter setup of our photoionization simulations. In Section 3, we present the major line luminosity radial profiles for various SEDs. The same line emission versus radius relations, but for different gas densities, are presented in Section 4. Finally, the discussion and conclusions of our work are described in Sections 5 and 6.

\section{THE MODEL AND ITS PARAMETERS}

We consider the continuous distribution of optically thick spherical clouds above an accretion disk, placed at different radial distances extending from the BLR $\left(\sim 10^{-2} \mathrm{pc}\right)$ to the NLR $\left(\sim 10^{3} \mathrm{pc}\right)$. Each cloud at radial distance $r$ from the nucleus represents the gas in the emission region described by the parameters: hydrogen number density, $n_{\mathrm{H}}\left[\mathrm{cm}^{-3}\right]$, dimensionless ionization parameter, $U$, total hydrogen column density, $N_{\mathrm{H}}\left[\mathrm{cm}^{-2}\right]$, and the chemical abundances. We note that, in recent years, there is growing evidence that the emitting and absorbing clouds are radiation pressure confined, and thus the total (radiation + gas) pressure inside the cloud is constant with stratification in matter density (Pier \& Voit 1995; Dopita et al. 2002; Groves et al. 2004a, 2004b; Różańska et al. 2006; Baskin et al. 2014; Stern et al. 2014; Adhikari et al. 2015; Stern et al. 2016). However, we have checked that the assumption that each cloud is in pressure equilibrium does not change the main conclusion of our paper; therefore, here we consider the constant-density clouds to keep the consistency with the NL93 formalism. The ionization parameter is defined as the ratio of the number of hydrogen-ionizing photons, $Q_{\mathrm{H}}\left[\mathrm{s}^{-1}\right]$, to the gas density of the cloud (Osterbrock \& Ferland 2006)

$$
U=\frac{Q_{\mathrm{H}}}{4 \pi r^{2} n_{\mathrm{H}} c}
$$

where $c$ is the velocity of light.

These clouds are illuminated by the radiation of different spectral shapes shown in Figure 1. To consider various types of AGNs, we used the SED of the Sy1.5 galaxy Mrk 509 as measured in multiwavelength observation campaigns (Kaastra et al. 2011) and the Syl galaxy NGC 5548 (Mehdipour et al. 2015). The SED of Mrk 509 is dominated by soft X-ray photons below $1 \mathrm{keV}$, whereas the SED of NGC 5548 is dominated by harder photons above $2 \mathrm{keV}$. To represent the SED of an NLSy1 we use the galaxy PMN J0948+0022 (D'Ammando et al. 2015), shown as the magenta dashed line. The SED of NLSy1 galaxy PMN J0948+0022 has no pronounced emission around $\sim 0.1 \mathrm{keV}$; rather, it has an excess of harder photons. We also consider the spectral shape produced with the Band function $f(E)$ (Band et al. 1993) as another typical active galaxy. The Band function combines two power laws smoothly and provides the possibility of creating different shapes of spectra by allowing us to change the parameters in the following expression:

$$
\begin{aligned}
& f(E)=A\left[\frac{E}{100}\right]^{\alpha} e^{\left[\frac{-2(E+\alpha)}{E_{\mathrm{p}}}\right]}, \quad \text { for } E<\frac{(\alpha-\beta) E_{\mathrm{p}}}{(2+\beta)} \\
& =A\left[\frac{(\alpha-\beta) E_{\mathrm{p}}}{100(2+\alpha)}\right]^{(\alpha-\beta)}\left(\frac{E}{100}\right)^{\beta} e^{(\beta-\alpha)}, \\
& \text { for } E \geqslant \frac{(\alpha-\beta) E_{\mathrm{p}}}{(2+\beta)} \text {, }
\end{aligned}
$$

where $\alpha$ and $\beta$ are the slopes of the first and second power law, respectively. $E_{\mathrm{p}}$ is the peak energy, where the two power laws combine smoothly. For producing the Band SED shape, we choose the slope of the first power law $\alpha=0.51$, which cuts 


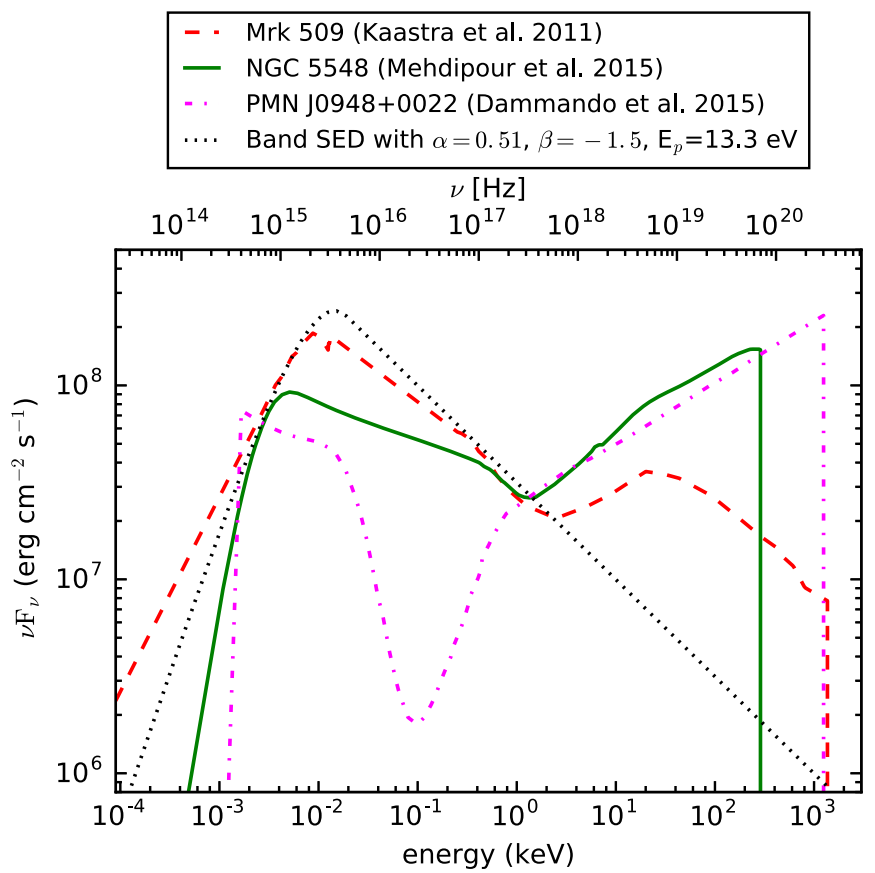

Figure 1. Shapes of the broadband spectra used in our photoionization calculations. All SEDs are normalized to $L=10^{45} \mathrm{erg} \mathrm{s}^{-1}$ and represent the radiation illuminating the cloud at $r=0.1 \mathrm{pc}$. The dashed line shows Mrk 509, while the solid line describes NGC 5548. The SED of the NLSy1 PMN J0948 +0022 is presented as the dot-dashed line, and the SED produced with the Band function is shown by the dotted line. See Section 2 for parameters and references.

off exponentially at $13.3 \mathrm{eV}$, and the second power law with slope $\beta=-1.5$. These SEDs are chosen for two reasons: (i) they are recently constrained by combining the data from multiwavelength observations (except the Band SED), and (ii) they represent different possible SEDs that an AGN can have in general.

For deriving the luminosities of the emission lines corresponding to each individual portion of gas at the given distance, we make simulations with the photoionization CLOUDY code (version c13.03; Ferland et al. 2013). We use the luminosity case in CLOUDY with an assumed source luminosity of $L=10^{45} \mathrm{erg} \mathrm{s}^{-1}$. Thus, the ionization parameter $U$ for each cloud is computed by the code itself. The shape of the continuum is given by points using the interpolate command (for details see CLOUDY documentation files ${ }^{4}$ ). We employed the profiles of the cloud parameters as a function of $r$ as described by NL93:

$$
n_{\mathrm{H}} \propto r^{-3 / 2}, \quad N_{\mathrm{H}} \propto r^{-1} .
$$

To investigate the effect of the SED on the resulting line luminosities, the normalization of parameters in Equation (3) is chosen at $r=0.1 \mathrm{pc}, n_{\mathrm{H}}=10^{9.4} \mathrm{~cm}^{-3}$ and $N_{\mathrm{H}}=10^{23.4} \mathrm{~cm}^{-2}$, following the canonical NL93 model. With this choice of normalization, the values of $n_{\mathrm{H}}$ and $N_{\mathrm{H}}$ of the cloud at the closest distance, $r=10^{-2} \mathrm{pc}$, are $10^{10.9} \mathrm{~cm}^{-3}$ and $10^{24.4} \mathrm{~cm}^{-2}$, respectively. The most distant cloud, at $r=10^{3} \mathrm{pc}$, has values of $10^{3.4} \mathrm{~cm}^{-3}$ and $10^{19.4} \mathrm{~cm}^{-2}$, respectively. As justified in NL93, these numbers are reasonable for simulating the BLR-NLR system of an AGN with a bolometric luminosity of $10^{45} \mathrm{erg} \mathrm{s}^{-1}$. The choice of the sublimation

\footnotetext{
http://www.nublado.org/
}

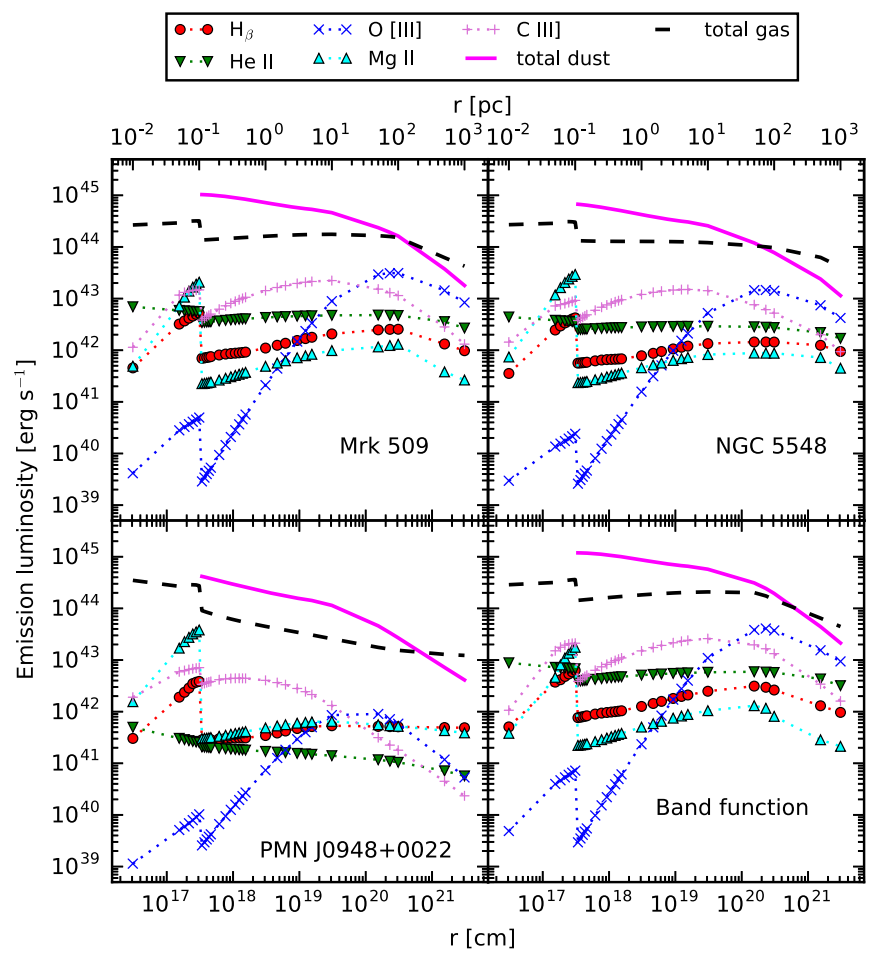

Figure 2. Line emission vs. radius for various lines: $\mathrm{H} \beta$ (red circles), He II (green downward-pointing triangles), [O III] (blue crosses), Mg II (cyan upward-pointing triangles), and C III] (magenta plus signs) shown as a function of radius from the illuminating source. For all panels $n_{\mathrm{H}}=10^{9.4} \mathrm{~cm}^{-3}$ at $r=0.1 \mathrm{pc}$. Total dust emission (magenta solid line) and the total gas emission (black dashed line) are shown for clarity. Upper left panel: results for spectral shapes of Mrk 509; upper right panel: NGC 5548; lower left panel: NLSy1 PMN J0948+0022; lower right panel: Band function.

radius at $0.1 \mathrm{pc}$ is not obvious, since it depends on the size and the grain composition (Netzer 2013). However, for the purpose of understanding the global properties of the line emission versus radius, this assumption is reasonable, and it was also used by NL93.

We used the CLOUDY default solar composition for clouds located at $r \leqslant 0.1 \mathrm{pc}$, and for more distant clouds at $r>0.1 \mathrm{pc}$ the interstellar medium (ISM) composition with dust grains is used. This includes the graphite and silicate component appropriate for the ISM of our Galaxy and is fully taken into account by the CLOUDY code.

To investigate the role of the cloud density on our results, we change the normalization to give different values of the gas density number at the sublimation radius. We consider the following four different number densities: $n_{\mathrm{H}}=10^{7}, 10^{10}$, $10^{11}, 10^{11.5} \mathrm{~cm}^{-3}$ at $r=0.1 \mathrm{pc}$, while keeping the other parameters the same.

\section{LINE EMISSION VERSUS RADIUS FOR VARIOUS SEDs}

In this section we set the density normalization as in NL93, so that $n_{\mathrm{H}}=10^{9.4} \mathrm{~cm}^{-3}$ at $r=0.1 \mathrm{pc}$. The emission-line luminosity radial profiles for the lines $\mathrm{H} \beta$ (red circles), He II (green downward-pointing triangles), Mg II (cyan upwardpointing triangles), $\mathrm{C} \mathrm{III]} \mathrm{(magenta} \mathrm{plus} \mathrm{signs),} \mathrm{and} \mathrm{[O} \mathrm{III]} \mathrm{(blue}$ crosses) are shown in Figure 2. The upper panels of the figure show the line luminosities for Mrk 509 (left) and NGC 5548 (right). In the lower panels, results for PMN J0948+0022 (left) and the Band function (right) are presented. 
The nature of the line emission versus radius is similar for Mrk 509, NGC 5548, and the Band function, independently of the shape of the illuminating radiation. For all the considered lines, the radial emission profiles display the strong suppression (by a factor of $\sim$ few to a few orders of magnitude) of the line luminosity in a region close to the sublimation radius. The suppression of the emission can also be noticed in the profile of the total gas emission (black dashed lines in the figure). These results are in agreement with the result of NL93 performed for only the one standard AGN SED (Mathews \& Ferland 1987) used by those authors. However, the radial luminosity profile of the He II line is only weakly suppressed by a factor of $\sim 3$ with the inclusion of dust. This is different from the case of NL93, where the He II emission goes down by roughly an order of magnitude.

A considerably different radial emission profile for the semiforbidden line $\mathrm{C} \mathrm{III]} \mathrm{and} \mathrm{forbidden} \mathrm{line} \mathrm{[O} \mathrm{III]} \mathrm{is} \mathrm{obtained} \mathrm{in} \mathrm{the}$ case of the SED of NLSy1 PMN J0948+0022. The C III] line luminosity decreases steeply with increasing radius, showing a different behavior from the other SEDs, where it increases in a region between $0.1 \mathrm{pc}<r<10 \mathrm{pc}$. As can be seen from Figure 2 (lower left panel), the [O III] emission is not significant in a region corresponding to the NLR $(r \sim 10-100 \mathrm{pc})$, which is different from the other SEDs, where an excess of [O III] emission is seen at those distances. Interestingly, this result is consistent with the SDSS spectrum of PMN J0948+0022, where [O III] emission is not present (Tanaka et al. 2014, see their Figure 2, lower panel). Finally, our model shows that He II emission in this object is not significantly affected by the presence of dust. Rather, it is fairly constant close to $0.1 \mathrm{pc}$ and decreases slowly as a function of radial distance. The differences in line emission of $\mathrm{C} \mathrm{III],} \mathrm{[O} \mathrm{III],} \mathrm{and} \mathrm{He} \mathrm{II} \mathrm{from}$ the other SEDs considered in our study are connected with the fact that the PMN J0948+0022 SED has fewer photons in the extreme-UV and soft X-ray band. This significantly smaller number of hydrogen-ionizing photons causes the drastic drop, by two orders of magnitude, in lines emitted in the lowerdensity dusty medium on the larger radii.

Despite these differences, the effect of dust on the line suppression is clearly visible in the case of PMN J0948+0022, and it is strongest for the high-luminosity $\mathrm{H} \beta$ and $\mathrm{Mg}$ II lines. The general conclusion is that for gas with physical conditions and parameters given by NL93, the apparent gap in the line emission cannot be removed by changing the shape of the illuminating continuum. The differences in the [O III], C III], and He II radial luminosity profiles for the NLSy1 SED are not sufficient to explain the origin of the ILR.

\section{ROLE OF THE GAS DENSITY}

High local densities in the BLR $\left(n_{\mathrm{H}} \sim 10^{11}-10^{11.5} \mathrm{~cm}^{-3}\right)$ have been deduced from the study of UV Fe II emission of an extreme NLSy1 object, I Zw 1 (Bruhweiler \& Verner 2008), and in the quasars LBQS 2113-4538 (Hryniewicz et al. 2014), CTS C30.10 (Modzelewska et al. 2014), and HE 0435-4312 (Sredzinska et al. 2016). Additionally, a density of $10^{10.25} \mathrm{~cm}^{-3}$ was found from the study of UV intrinsic absorbers of two NLSy1 galaxies (Leighly 2004). Finally, from photoionization calculations, Różańska et al. (2014) found the density of an intrinsic absorbing cloud in the bright quasar HS $1603+3820$ to be of the order of $n_{\mathrm{H}} \sim 10^{10}-10^{12} \mathrm{~cm}^{-3}$. On the basis of those results, we increased the normalization of the gas density at $r=0.1 \mathrm{pc}$, to be $n_{\mathrm{H}}=10^{11.5} \mathrm{~cm}^{-3}$ in our model. Note that the

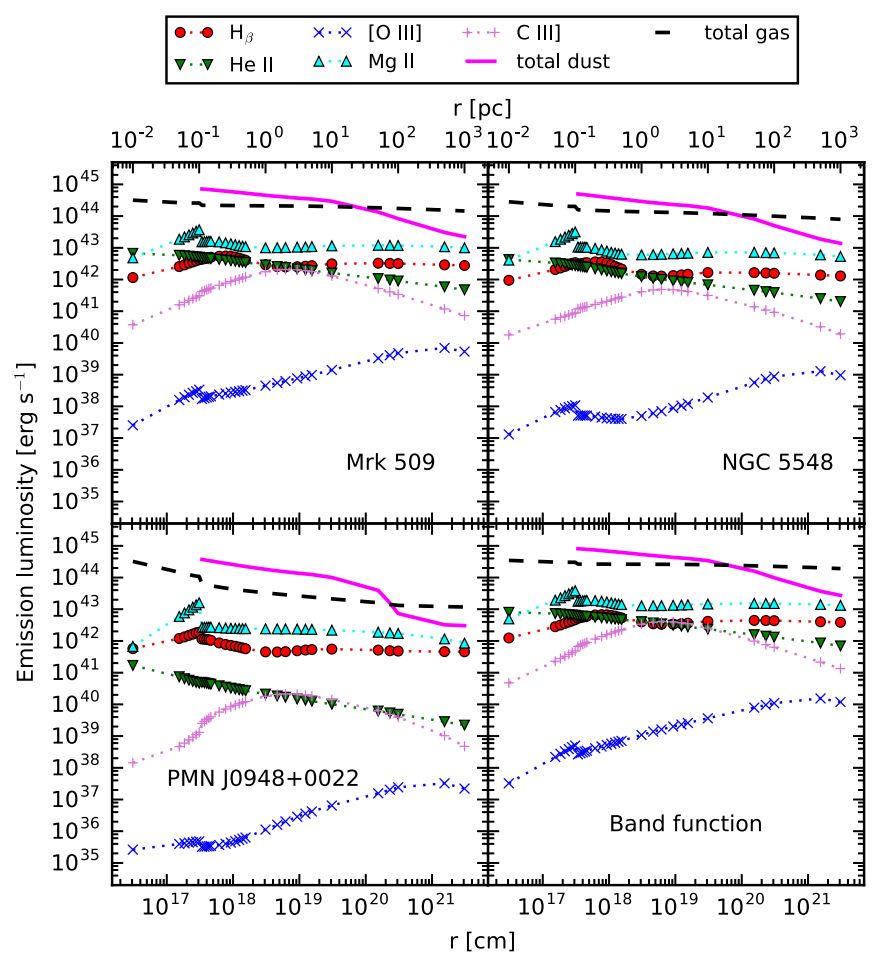

Figure 3. Same as Figure 2, but for density $n_{\mathrm{H}}=10^{11.5} \mathrm{~cm}^{-3}$ at the sublimation radius, $r=0.1 \mathrm{pc}$.

ionization parameter changes inversely with $n_{\mathrm{H}}$ as given in Equation (1), but all other parameters have the same values as described in Section 2.

The resulting emission profiles of the five major lines are shown in Figure 3 for the four SEDs. The meaning of lines and points is exactly the same as in Figure 2. The $\mathrm{H} \beta$, He II, and $\mathrm{C}$ III] emission lines behave differently from the lower-density normalization case. The presence of dust does not suppress $\mathrm{H} \beta$ and $\mathrm{C}$ III] line emission; rather, it is enhanced by the factor of $\sim 1.5$ at $0.1<r<0.3 \mathrm{pc}$, in the case of the Mrk 509, NGC 5548, and Band function SEDs. For the SED of NLSy1 PMN J0948+0022 C III] emission increases by a factor of $\sim 3$ at $0.1<r<0.3 \mathrm{pc}$, whereas $\mathrm{H} \beta$ emission decreases slightly with the distance, but there is no large jump at the sublimation radius, as was found in the lower-density case. Only the $\mathrm{Mg}$ II line displays a jump at $0.1 \mathrm{pc}$, but it is much less prominent than in the lower-density case. In all other cases we observe the lack of the line suppression for all shapes of SED.

Our result clearly shows that the strong suppression in the line emission at the sublimation radius for lower-density normalization disappears at higher density. Therefore, intermediate-line emission can be produced at radial distances $r \sim$ $0.1-1 \mathrm{pc}$. Note that the gas emission also does not display any jump for the high-density case. The strong ILR emission found by Mason et al. (1996), Crenshaw \& Kraemer (2007), and Crenshaw et al. (2009) can be produced if the clouds are two orders of magnitude denser than those considered by NL93. Our results corroborate with the speculation made by Landt et al. (2014), where they discuss the possible role of highdensity gas for the production of a smooth BLR. Furthermore, if the ILR is located at distances predicted by our model, we can estimate the expected reverberation mapping lag. Within the framework of our model, the ILR lag would be of the order of 100-1000 lt-day. 
The physical reason for the density dependence is connected with the value of the column density at which the ionization front is located. Below, we explain this in the example of the hydrogen $\mathrm{H} \beta$ line. Let us assume simple ground-state hydrogen photoionization and set the ionization balance equation as

$$
n_{\mathrm{p}} n_{\mathrm{e}} l \alpha_{\mathrm{B}}(T)=\phi_{\mathrm{H}},
$$

(Osterbrock \& Ferland 2006). The first two terms are the proton and electron density in the $\mathrm{H}^{+}$layer of the cloud, $l[\mathrm{~cm}]$ is the thickness of the $\mathrm{H}^{+}$layer, and $\alpha_{\mathrm{B}}(T)\left[\mathrm{cm}^{3} \mathrm{~s}^{-1}\right]$ is the case $\mathrm{B}$ recombination coefficient. The physical interpretation is that the flux of hydrogen-ionizing photons incident on the cloud, $\phi_{\mathrm{H}}$ $\left[\mathrm{cm}^{-2} \mathrm{~s}^{-1}\right]$, equals the number of hydrogen recombinations that occur over the thickness $l$.

The hydrogen column density across the $\mathrm{H}^{+}$layer is then

$$
N_{\mathrm{H}^{+}} \equiv n_{\mathrm{p}} l=\frac{\phi_{\mathrm{H}}}{n_{\mathrm{e}} \alpha_{\mathrm{B}}(T)}=U c \alpha_{\mathrm{B}}(T)^{-1} .
$$

The gas column density $N_{\mathrm{H}^{+}}$and the resulting line and continuum optical depths all scale with the ionization parameter. At very high values of $U$, the whole cloud is ionized, and the line emission comes from the whole volume, in this case limited by the fixed adopted total hydrogen column, $N_{\mathrm{H}}$. As the cloud density increases, the ionization parameter decreases, the ionization front forms, and the cloud consists of two zones. The line emission comes from the first zone, and only the dust in this zone competes with the gas for the photons. The high-density clouds studied here have smaller column densities and optical depths of the $\mathrm{H}^{+}$layer, since the ionization parameter is lower. This is clearly demonstrated in Figure 4, which shows that the $\mathrm{H}^{+}-\mathrm{H}^{0}$ ionization front moves toward smaller cloud thickness with increasing number density of the cloud. For smaller values of $U$, the dust absorption decreases, so there is less suppression of the emission due to the presence of dust. In other words, the less dense clouds have much larger thickness of the $\mathrm{H}+$ layer and larger dust column density in the zone with abundant photons, and therefore the dust absorption is significant.

Equation (5) can be used for the quantitative estimation of threshold $U$, below which the gas opacity for incident photons dominates over opacity of dust. Taking recombination coefficient $\alpha_{\mathrm{B}}\left(\right.$ at $\left.10^{4} \mathrm{~K}\right)=2.6 \times 10^{-13} \mathrm{~cm}^{3} \mathrm{~s}^{-1}$ gives the column density $N_{\mathrm{H}^{+}} \sim 10^{23} \mathrm{U}$. Therefore, the dust optical depth for UV photons is $\tau_{\text {dust }} \cong N_{\mathrm{H}^{+}} / 10^{21}=100 U$ (for details see Güver \& Özel 2009). For $U=0.1, \tau_{\text {dust }}=10$ at the edge of the $\mathrm{H}^{+}$region. This implies that the width of the $\mathrm{H}^{+}$layer where gas actively absorbs and emits is only $1 / 10$ of the total layer. In other words, increasing the density by a factor $>10$ would mean $U<0.01$, which implies $\tau_{\text {dust }}<1$. This means that the gas opacity always dominates for higher densities and it does not matter whether the gas is dusty or not, and therefore no suppression of the line emission is physically possible.

It is worth mentioning the conclusion of Ferland \& Netzer (1983), which states that for low-ionization nuclear emissionline region (LINERs), if the line emission comes from the photoionized gas, $U$ is $\leqslant 10^{-3}$. This is less than the threshold $U$ corresponding to higher density $\left(10^{11.5} \mathrm{~cm}^{-3}\right)$ implied by our result, where the gas opacity is always dominant over the opacity of dust. Thus, our result clearly indicates that LINERs should also exhibit the ILR. We note that the presence of the
ILR in LINERs is also discussed by Balmaverde et al. (2016), where they analyzed 33 LINERs (bona fide AGNs) and Seyfert galaxies from the optical spectroscopic Palomar survey observed by HST/STIS. However, the density of the outer portions of the ILR claimed by those authors is about three orders of magnitude less (i.e., $10^{4}-10^{5} \mathrm{~cm}^{-3}$ ) than what our model predicts. Most probably this is due to the fact that LINERs are much fainter than the objects considered in this paper.

\section{DISCUSSION}

\subsection{Individual Line Behavior}

Besides the general trend presented above, in some cases we note exceptional line behavior. For instance, Mg II (cyan upward-pointing triangles in Figures 2, and 3) line emissivity does not depend on the shape of the SED. All SEDs considered by us produce the emissivity jump at the sublimation radius, but this jump is two orders of magnitude higher when the cloud density is lower, due to the physical reason given above, but for the case of the magnesium ionization front.

The density influence on the suppression of the line luminosity jump at the sublimation radius is best seen in the case of $\mathrm{H} \beta$. For all continuum shapes the strong jump in emission (order of magnitude) is present for the canonical density used by NL93, $n_{\mathrm{H}}=10^{9.4} \mathrm{~cm}^{-3}$. This jump is not seen at all for much denser clouds.

Note that the dust influence on the emission jump is much stronger in the case of the $\mathrm{Mg}$ II line than for the $\mathrm{H} \beta$ line. This difference is due to the fact that the $\mathrm{H} \beta$ line forms in deeper parts of a cloud than the Mg II line. In the presence of dust, the radiation reaching these depths is harder since the dust opacity selectively removes lower-energy photons, allowing harder photons to reach the region where $\mathrm{H} \beta$ is formed. As a result, the $\mathrm{H} \beta$ forming region is warmer and more ionized, making the line stronger. Moreover, the dust opacity is smaller at the wavelength of $\mathrm{H} \beta$ than $\mathrm{Mg}$ II, so $\mathrm{H} \beta$ is absorbed less than $\mathrm{Mg}$ II. This implies that the contribution of the NLR to the overall line shape is expected to be lower in $\mathrm{Mg}$ II than in $\mathrm{H} \beta$.

The He II line emission versus radius from our simulations shows that the luminosity jump is at least one order of magnitude lower than the jump reported by NL93 for the same line. Our simulations show that this jump is much smaller, only a factor of two, for three Sy1 SEDs, and completely disappears in the case of the NLSy1 continuum. Those differences may be caused by the different numerical code used by those authors. Nevertheless, with the high-density case, He II line suppression is not present for all considered shapes of radiation.

For both densities, the C III] line strongly depends on the SED shape. With the NLSy1 PMN J0948+0022 SED and the dust presence, line luminosity slowly decreases with distance by $\sim$ few factors, and the maximum line emission always occurs at $r<0.1 \mathrm{pc}$. In the case of the Sy1 SEDs, the line luminosity peaks at $r \sim 10 \mathrm{pc}$, with clear suppression at the sublimation radius for the low-density case.

The [O III] emission (blue crosses in Figures 2 and 3) reaches a maximum farther outside the sublimation radius. For the high-density case, this line is very weak in comparison with other lines. This is in agreement with the fact that forbidden lines originate from low-density gas. Only for low-density case is there enhanced [O III] emission in a region close to the NLR $(r \sim 10-100 \mathrm{pc})$. We expect that such a line will never be 
observed in the ILR. Indeed, the initial claim of the [O III] variability was not supported by the careful analysis of the data (Barth \& Bentz 2016). Besides the forbidden [O III] line, our results strongly support the presence of an ILR where the intermediate-velocity lines are expected.

\subsection{Apparent Gap Suppression}

To investigate the role of density in the formation of the ILR, we calculated our model for several values of density normalizations: $n_{\mathrm{H}}=10^{7.0}, 10^{9.4}, 10^{10.0}, 10^{11.0}, 10^{11.5} \mathrm{~cm}^{-3}$, keeping other parameters the same. Figure 5 shows the dependence of line luminosity radial profiles on the density normalization for two representative SEDs: Mrk 509 (left panels) and PMN J0948+0022 (right panels), for all lines considered by us.

For the rare clouds, the luminosity of some lines decreases many orders of magnitude. Those lines would not be observed due to the sensitivity of the telescopes. Therefore, we mark the lower limit on the line luminosity above which the line could be visible by the horizontal dashed line in each panel. We estimated this limit by assuming a Gaussian spectral profile of the emission lines on top of the underlying continuum given by our SEDs. For this calculation, the spectral line width is set to the value corresponding to Keplerian velocity at $1 \mathrm{pc}$ ( $\sim$ ILR radii), for a BH mass $\sim 10^{8} M_{\odot}$. This is a rather hard lower limit on the observable line luminosities since the simulated isotropic luminosities should be at least 10 times higher (assuming 100\% covering factor). Nevertheless, simulated line luminosities presented in Figure 4 fall below this limit in some cases, and those lines have no chance to be detected.

Note that for a given SED there is a preferred value of density for which the line emission is the greatest. For example, the $\mathrm{H} \beta$ luminosity increases with the density normalization and is a maximum for $n_{\mathrm{H}}=10^{11.5} \mathrm{~cm}^{-3}$ at distances $0.1 \mathrm{pc}$ $<r<0.4 \mathrm{pc}$. The He II luminosity peaks at $r<0.1 \mathrm{pc}$, and the maximum emission occurs for the lower density, whose value depends on the SED shape. In the case of PMN J0948 +0022 the luminosity peak is lower by an order of magnitude than for the Mrk 509 SED and occurs for density $n_{\mathrm{H}}=10^{7} \mathrm{~cm}^{-3}$. This is in agreement with the Locally Optimized Cloud model of Baldwin et al. (1995).

The radial distances at which the $\mathrm{H} \beta$ and $\mathrm{He}$ II emissions are the strongest are in agreement with the results inferred from the reverberation studies of the BLR in AGNs. The radial stratification with ionization potential of the species producing the line has been observed (i.e., Clavel et al. 1991; Peterson \& Wandel 1999), showing that the He II line always originates at distances closer by a factor of three/four to the nucleus than the $\mathrm{H}$ line. In addition, the distances for which the luminosities of $\mathrm{Mg}$ II and [O III] peak are consistent with the reverberation mapping studies.

For the Mrk 509 SED, the emission, with density normalization $n_{\mathrm{H}}=10^{7} \mathrm{~cm}^{-3}$ at $0.1 \mathrm{pc}$ is insignificant for all considered lines. However, the [O III] emission is the strongest for the PMN J0948+0022 SED with the lowest density, which agrees with the prediction that forbidden lines are formed in low-density gas. For both SEDs, the semi-forbidden line C III] has a maximum emission at intermediate densities, i.e., $n_{\mathrm{H}}=10^{9.4} \mathrm{~cm}^{-3}$ at $0.1 \mathrm{pc}$.

Despite the different distances where the line luminosities peak, the strong jump in the emission profiles disappears in almost all cases for both shapes of continuum, when density

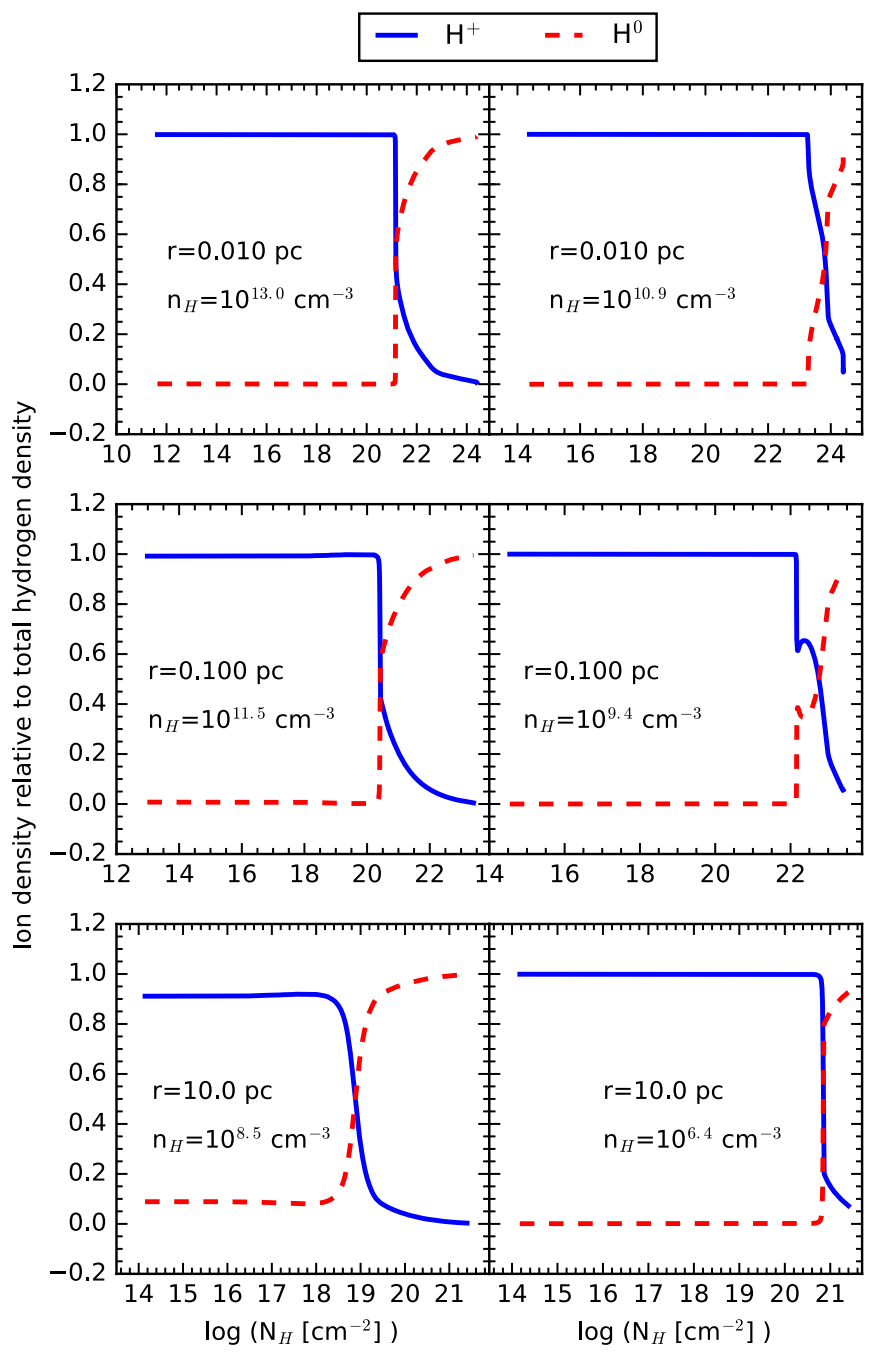

Figure 4. Ionized $\left(\mathrm{H}^{+}\right)$and neutral $\left(\mathrm{H}^{0}\right)$ hydrogen densities relative to the total cloud denesity $n_{\mathrm{H}}$, as a function of column density across the cloud illuminated by the SED of Mrk 509. The left and right panels show the ion densities for $n_{\mathrm{H}}=10^{11.5}$ and $10^{9.4} \mathrm{~cm}^{-3}$ at $r=0.1 \mathrm{pc}$, respectively. Values of $n_{\mathrm{H}}$ change with $r$ as in Equation (3) and are given in each panel together with the cloud location.

normalization increases. The lack of this jump clearly means that there will not be a gap between the BLR and NLR. This naturally explains the origin of the ILR.

\subsection{The Connection with an Accretion Disk}

It is widely believed that broad-line emission clouds may be connected with the wind from an accretion disk atmosphere (Gaskell 2009; Czerny \& Hryniewicz 2011). The upper atmospheric layers of the disk can be quite dense, with values up to $n_{\mathrm{H}} \sim 10^{14} \mathrm{~cm}^{-3}$, depending on the distance from the central black hole (Hryniewicz 2011; Różańska et al. 2014). These densities are derived by solving for the accretion disk vertical structure in hydrostatic and radiative equilibrium parameterized by the black hole mass, its spin, and the accretion rate (Różańska et al. 1999). We can properly derive the density at the disk photosphere, i.e., at the optical depth $\tau \sim 2 / 3$, assuming energy generation via viscosity, and diffusion approximation of the radiative transfer. Our calculations use the Rosseland mean opacity tables from Alexander et al. (1983) and Seaton et al. (1994). Those opacities are 


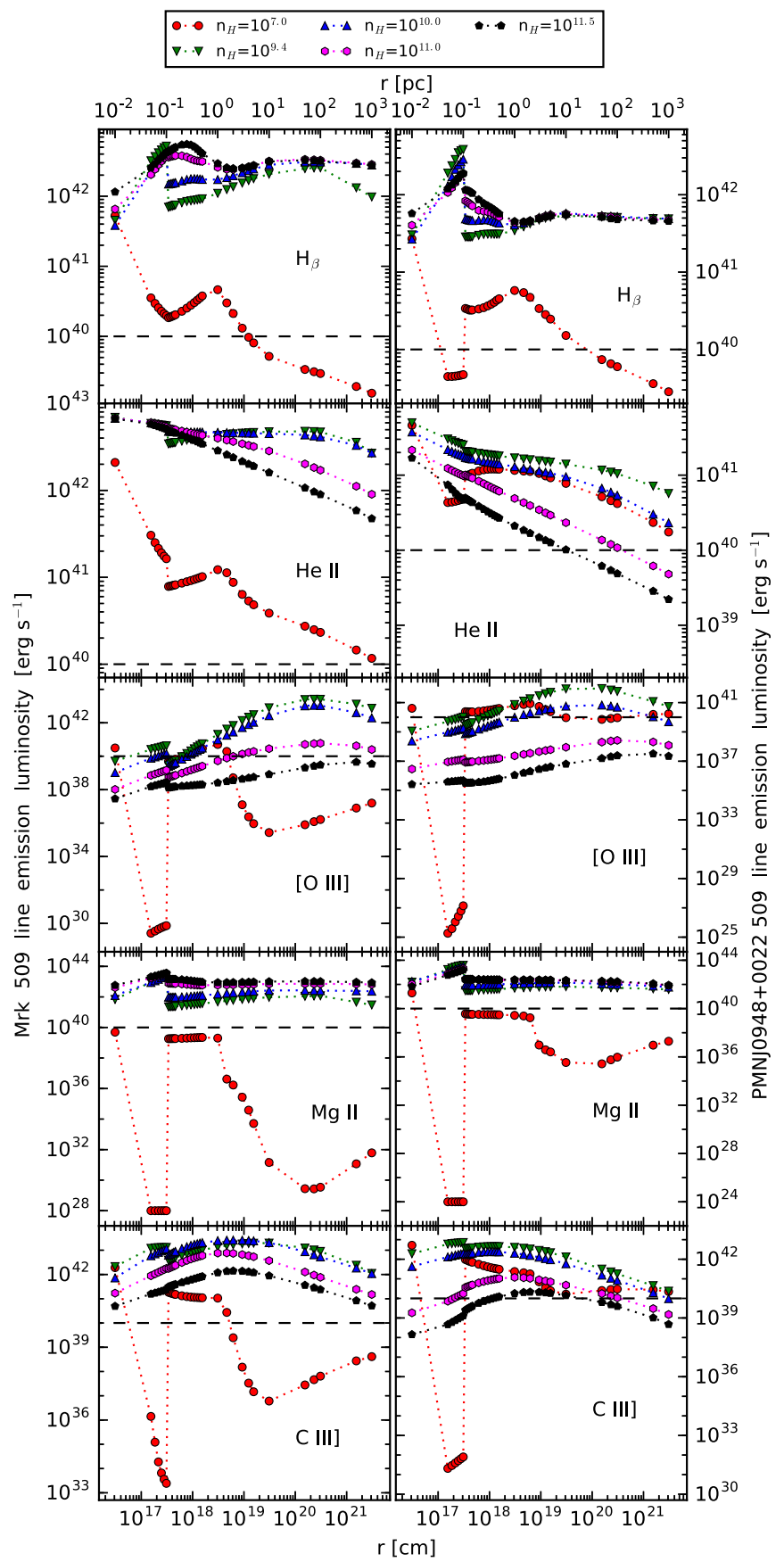

Figure 5. Line emission vs. radius for different densities: $n_{\mathrm{H}}=10^{7.0}$ (red circles), $10^{9.4}$ (green downward-pointing triangles), $10^{10.0}$ (blue upwardpointing triangles), $10^{11.0}$ (magenta hexagons), and $10^{11.5} \mathrm{~cm}^{-3}$ (black pentagons) at $r=0.1 \mathrm{pc}$. The left and right panels show the case of the Sy1.5 Mrk 509 and NLSy1 PMN J0948+0022 SEDs, respectively. Five major lines, $\mathrm{H} \beta$, He II, [O III], Mg II, and C III], are presented from top to bottom. The detection limit on line luminosity is shown by the horizontal black dashed line (see text for details).

crucial for calculating the disk density since the true absorption opacity can be an order of magnitude higher than electron scattering opacity, as shown by Różańska et al. (1999, see their Figures 4 and 7).

We take the density at $\tau=2 / 3$ as a representative of the initial density in the disk wind, which supplies matter to the BLR or intrinsic absorbers in an outflow. The model fully takes

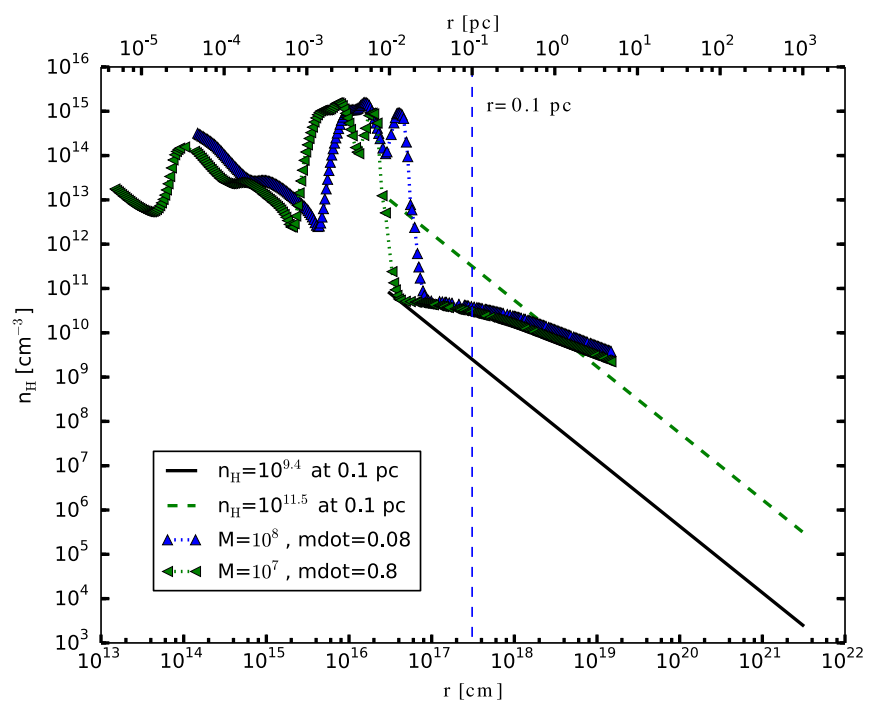

Figure 6. Accretion disk atmosphere density radial profiles. The vertical dashed line marks the position of the sublimation radius. Straight lines are plotted according to the power law given in Equation (3) by the first term for two different density normalizations marked in the figure. Two data sets are presented for expected masses and Eddington ratios derived from an assumed bolometric luminosity $\left(10^{45} \mathrm{erg} \mathrm{s}^{-1}\right.$; see text for details).

into account the radial transition from pressure-dominated regions to gas-dominated regions by assuming that the viscous torque is proportional to the total pressure (gas and radiation), and we use a realistic description of the opacities, including atomic transitions and the presence of dust. Nevertheless, the model should not be extended too far beyond a $\sim 1 \mathrm{pc}$ scale because there the self-gravity effects and the effects of the circumnuclear stellar cluster become important (Thompson et al. 2005).

The disk atmosphere radial density profile at $\tau=2 / 3$ is plotted in Figure 6. We used two test sets of parameters. Keeping the bolometric luminosity constant at $10^{45} \mathrm{erg} \mathrm{s}^{-1}$, we find the Eddington ratio for masses of $10^{7} M_{\odot}$ and $10^{8} M_{\odot}$. We can assume that our rescaled SEDs should be produced in AGNs with BH masses in this range. For the given assumptions and taking a radiative efficiency of 0.1 , we then derive the Eddington ratios corresponding to those masses: 0.8 and 0.08 , respectively. In addition, the density of the clouds used in our BLR-NLR simulations is drawn by straight lines according to Equation (3), for two normalizations at the sublimation radius: $n_{\mathrm{H}}=10^{9.4}$ and $10^{11.5} \mathrm{~cm}^{-3}$.

It is thus clearly seen that accretion disk atmospheres naturally produce high densities at the sublimation radius. All models show a strong change in density produced by heavyelement Rosseland mean opacities used in our calculations. Even if this bump is slightly inside the sublimation radius assumed by us, the density radial profiles show that high densities occur in the disk atmosphere, and such dense gas can give rise to the line-emitting regions.

On the other hand, the local density at the disk atmosphere does not depend on the Eddington ratio and is expected to be the same for the Sy1 and NLSy1 galaxies. Thus, the differentiation in the local density between those two types of objects must happen at the wind formation stage. Smooth wind outflow causes the decrease of the density with the distance measured along the wind stream line due to wind acceleration and the geometrical divergence of the wind line. However, if 
thermal instability operates in the wind and a colder/denser cloud forms, the evolution of the wind density becomes very complex and dependent on the heating and cooling efficiency. The difference between the wind developing in Sy1 and NLSy 1 may be due to the difference in the basic wind velocity. A given disk radius, measured in $\mathrm{cm}$ or $\mathrm{pc}$, corresponds to a larger value of the $r / R_{\text {Schw }}$ in NLSy1 than in Sy1, the corresponding Keplerian velocity is lower, and the wind velocity, which usually is of the same order, is also smaller, leaving perhaps more time for the development of the thermal instability in the wind. This would explain the higher density of the clouds in NLSy1 than in Sy1. However, detailed analysis of the timedependent wind model is certainly beyond the scope of the current paper.

\section{CONCLUSIONS}

Following the work of NL93, we performed numerical simulations of photoionized gas in AGN emission-line regions and derived the line luminosity radial profiles for various major lines: $\mathrm{H} \beta \lambda 4861.36$, He II $\lambda 1640.00, \mathrm{Mg}$ II $\lambda 2798.0, \mathrm{C}$ III] $\lambda 1909.00$, and [O III] $\lambda 5006.84$ originating at different distances from the central engine of the AGN.

On the basis of the line emission versus radius derived for four different SED shapes of incident radiation, and for different density normalizations at the sublimation radius, we find the following:

1. The presence or absence of the ILR is not determined by the spectral shape of the incident continuum. Different SEDs do produce considerably different behavior of the emission-line radial profiles, due to the different amount of extreme UV and soft X-ray photons in its broadband SED. However, these differences do not adequately explain why an intermediate-line emission is observed in some objects.

2. With higher density normalization, i.e., $n_{\mathrm{H}}=10^{11.5} \mathrm{~cm}^{-3}$ at $r=0.1 \mathrm{pc}$, we obtained flat luminosity line radial profiles for almost all lines and SEDs. Thus, the dust does not suppress the line emission, contrary to the result obtained by NL93. Our model potentially explains the existence of the ILR in some sources. If the density of the gas is high enough, emission lines of intermediate velocity width can be produced.

3. Such an ILR is predicted to be located at radial distances $r \sim 0.1-1 \mathrm{pc}$, and reverberation mapping lag expected by our model would be of the order of 100-1000 lt-day.

4. We demonstrated the dependence of line luminosity profiles on the density normalization. We found that the significant line emission in objects with a particular SED occurs at different densities, in agreement with reverberation mapping studies of $\mathrm{H} \beta, \mathrm{Mg}$ II, [O III], and $\mathrm{He}$ II lines.

5. We showed that dense clouds postulated by us can be potentially formed from an accretion disk atmosphere that is dense enough below the sublimation radius in the accretion disk. This work is another proof that the high densities occur in the BLR, which is in full agreement with many previous studies.

We are grateful to Jonathan Stern, the referee, for very helpful comments on the manuscript, and we thank Ari Laor and Jian-Min Wang for helpful discussion. This research was supported by Polish National Science Center grants No. 2011/
03/B/ST9/03281, 2015/17/B/ST9/03422, and by Ministry of Science and Higher Education grant W30/7.PR/2013. It received funding from the European Union Seventh Framework Program (FP7/2007-2013) under the grant agreement No. 312789. T.P.A. received funding from the NCAC PAS grant for PhD students. B.C. acknowledges the grant from the Foundation for Polish Science through the Master/Mistrz program 3/2012. G.J.F. thanks the Nicolaus Copernicus Astronomical Center for its hospitality and acknowledges support by NSF (1108928, 1109061, and 1412155), NASA (10-ATP10-0053, 10-ADAP10-0073, NNX12AH73G, and ATP13-0153), and STScI (HST-AR- 13245, GO-12560, HST-GO-12309, GO-13310.002-A, HST-AR-13914, and HST-AR-14286.001).

\section{REFERENCES}

Adhikari, T. P., Różańska, A., Sobolewska, M., \& Czerny, B. 2015, ApJ, 815,83

Alexander, D. R., Rypma, R. L., \& Johnson, H. R. 1983, ApJ, 272, 773

Baldwin, J., Ferland, G., Korista, K., \& Verner, D. 1995, ApJL, 455, L119

Baldwin, J. A., Ferland, G. J., Martin, P. G., et al. 1991, ApJ, 374, 580

Balmaverde, B., Capetti, A., Moisio, D., Baldi, R. D., \& Marconi, A. 2016, A\&A, 586, A48

Band, D., Matteson, J., Ford, L., et al. 1993, ApJ, 413, 281

Barth, A. J., \& Bentz, M. C. 2016, MNRAS, 458, L109

Baskin, A., Laor, A., \& Stern, J. 2014, MNRAS, 438, 604

Blandford, R. D., Netzer, H., \& Woltjer, L. 1990, Active Galactic Nuclei, ed. T. J.-L. Courvoisier \& M. Mayor, (New York: Springer)

Boroson, T. A., \& Green, R. F. 1992, ApJS, 80, 109

Brotherton, M. S., Wills, B. J., Francis, P. J., \& Steidel, C. C. 1994, ApJ, 430, 495

Bruhweiler, F., \& Verner, E. 2008, ApJ, 675, 83

Clavel, J., Reichert, G. A., Alloin, D., et al. 1991, ApJ, 366, 64

Crenshaw, D. M., \& Kraemer, S. B. 2007, ApJ, 659, 250

Crenshaw, D. M., Kraemer, S. B., Schmitt, H. R., et al. 2009, ApJ, 698, 281

Czerny, B., \& Hryniewicz, K. 2011, A\&A, 525, L8

Czerny, B., Modzelewska, J., Petrogalli, F., et al. 2015, AdSpR, 55, 1806

D’Ammando, F., Orienti, M., Finke, J., et al. 2015, MNRAS, 446, 2456

Davidson, K. 1972, ApJ, 171, 213

De Robertis, M. M., \& Osterbrock, D. E. 1984, ApJ, 286, 171

Dopita, M. A., Groves, B. A., Sutherland, R. S., Binette, L., \& Cecil, G. 2002, ApJ, 572, 753

Ferland, G., \& Netzer, H. 1979, ApJ, 229, 274

Ferland, G. J., \& Netzer, H. 1983, ApJ, 264, 105

Ferland, G. J., \& Osterbrock, D. E. 1986, ApJ, 300, 658

Ferland, G. J., Porter, R. L., van Hoof, P. A. M., et al. 2013, RMxAA, 49, 137

Gaskell, C. M. 2009, NewAR, 53, 140

Gaskell, C. M., Shields, G. A., \& Wampler, E. J. 1981, ApJ, 249, 443

Groves, B. A., Dopita, M. A., \& Sutherland, R. S. 2004a, ApJS, 153, 9

Groves, B. A., Dopita, M. A., \& Sutherland, R. S. 2004b, ApJS, 153, 75

Güver, T., \& Özel, F. 2009, MNRAS, 400, 2050

Hryniewicz, K. 2011, in Narrow-Line Seyfert 1 Galaxies and Their Place in the Universe (Trieste: PoS), 55

Hryniewicz, K., Czerny, B., Pych, W., et al. 2014, A\&A, 562, A34

Hu, C., Wang, J.-M., Ho, L. C., et al. 2008a, ApJL, 683, L115

Hu, C., Wang, J.-M., Ho, L. C., et al. 2008b, ApJ, 687, 78

Kaastra, J. S., Petrucci, P.-O., Cappi, M., et al. 2011, A\&A, 534, A36

Koshida, S., Takeo, M., Yoshii, Y., et al. 2014, ApJ, 788, 159

Krolik, J. H., McKee, C. F., \& Tarter, C. B. 1981, ApJ, 249, 422

Landt, H., Ward, M. J., Elvis, M., \& Karovska, M. 2014, MNRAS, 439, 1051

Leighly, K. M. 2004, ApJ, 611, 125

Li, Z., Zhou, H., Hao, L., et al. 2015, ApJ, 812, 99

Mason, K. O., Puchnarewicz, E. M., \& Jones, L. R. 1996, MNRAS, 283, L26

Mathews, W. G., \& Ferland, G. J. 1987, ApJ, 323, 456

Mehdipour, M., Kaastra, J. S., Kriss, G. A., et al. 2015, A\&A, 575, A22

Modzelewska, J., Czerny, B., Hryniewicz, K., et al. 2014, A\&A, 570, A53

Nenkova, M., Sirocky, M. M., Nikutta, R., Ivezić, Ž., \& Elitzur, M. 2008, ApJ, 685,160

Netzer, H. 2013, The Physics and Evolution of Active Galactic Nuclei (Cambridge, UK: Cambridge Univ. Press)

Netzer, H., \& Laor, A. 1993, ApJL, 404, L51 
Osterbrock, D. E., \& Ferland, G. J. 2006, Astrophysics of Gaseous Nebulae and Active Galactic Nuclei (Mill Valley, CA: Univ. Science Books)

Peterson, B. M., \& Wandel, A. 1999, ApJL, 521, L95

Pier, E. A., \& Voit, G. M. 1995, ApJ, 450, 628

Rees, M. J., Netzer, H., \& Ferland, G. J. 1989, ApJ, 347, 640

Różańska, A., Czerny, B., Życki, P. T., \& Pojmański, G. 1999, MNRAS, 305,481

Różańska, A., Goosmann, R., Dumont, A.-. M., \& Czerny, B. 2006, A\&A, 452, 1

Różańska, A., Nikołajuk, M., Czerny, B., et al. 2014, NewA, 28, 70

Seaton, M. J., Yan, Y., Mihalas, D., \& Pradhan, A. K. 1994, MNRAS, 266,805
Sredzinska, J., Czerny, B., Bilicki, M., et al. 2016, arXiv:1606.05130

Stern, J., Faucher-Giguère, C.-A., Zakamska, N. L., \& Hennawi, J. F. 2016, ApJ, 819, 130

Stern, J., Laor, A., \& Baskin, A. 2014, MNRAS, 438, 901

Suganuma, M., Yoshii, Y., Kobayashi, Y., et al. 2006, ApJ, 639, 46

Tanaka, M., Morokuma, T., Itoh, R., et al. 2014, ApJL, 793, L26

Thompson, T. A., Quataert, E., \& Murray, N. 2005, ApJ, 630, 167

van Hoof, P. A. M., Weingartner, J. C., Martin, P. G., Volk, K., \& Ferland, G. J. 2004, MNRAS, 350, 1330

Wills, B. J., Netzer, H., Brotherton, M. S., et al. 1993, ApJ, 410, 534

Zhu, L., Zhang, S. N., \& Tang, S. 2009, ApJ, 700, 1173 